\title{
Nanomechanics of collagen microfibrils
}

\author{
Simone Vesentini \\ Alberto Redaelli \\ Alfonso Gautieri
}

Biomechanics Group, Department of Electronics, Information and Bioengineering, Politecnico of Milan, Milan, Italy

\section{Corresponding author:}

Alfonso Gautieri

Biomechanics Group, Department of Electronics, Information and Bioengineering

Politecnico of Milan

P.zza Leonardo da Vinci, 32

20133 Milano, Italy

e-mail: alfonso.gautieri@polimi.it

\section{Summary}

Collagen constitutes one third of the human proteome, providing mechanical stability, elasticity and strength to organisms and is thus the prime construction material in biology. Collagen is also the dominating material in the extracellular matrix where its stiffness controls cell differentiation, growth and pathology. We use atomistic-based hierarchical multiscale modeling to describe this complex biological material from the bottom up. This includes the use and development of large-scale computational modeling tools to investigate several aspects related to collagen-based tissues, including source of visco-elasticity and deformation mechanisms at the nanoscale level. The key innovation of this research is that until now, collagen materials have primarily been described at macroscopic scales, without explicitly understanding the mechanical contributions at the molecular and fibrillar levels. The major impact of this research will be the development of fundamental models of collagenous tissues, important to the design of new scaffolding biomaterials for regenerative medicine as well as for the understanding of collagen-related diseases.

KEY WORDS: atomistic simulations, biomechanics, collagen, fibril, nanomechanics.

\section{Introduction}

The word collagen originates from the Greek word kó $\lambda \lambda a$ (kolla, meaning "glue") due to its ancient use as a glue source - obtained though the boiling of animal skin and collagen-rich tissues from Egyptians about 4,000 years ago'. In a broader sense, collagen could be seen as the "glue" of our body, holding it together by providing elasticity and strength to several tissues where a mechanical functions is needed, such as skin, cartilage, tendons and bones ${ }^{2,3}$.

From the structural point of view, collagen-rich tissues are built with the collagen fibril as fundamental building block. These fibrils have a diameter in the range of 30 to $500 \mathrm{~nm}$, a length up to the millimeter range and are assembled in complex hierarchical assemblies, whose structure depend on the particular tissue (Fig. 1).

At the lowest hierarchical level there is the collagen molecule (also called tropocollagen), that is a rod-like molecule with a length of about $300 \mathrm{~nm}$ and a diameter of about $1.5 \mathrm{~nm}$, made of three separate chains folded in the characteristic triple helical configuration 4,5 . According to the Hodge-Petruska model ${ }^{6}$ a structural model of collagen fibrils proposed in 1964 molecules in a fibril are deposited side by side and parallel but staggered with respect to each oth-

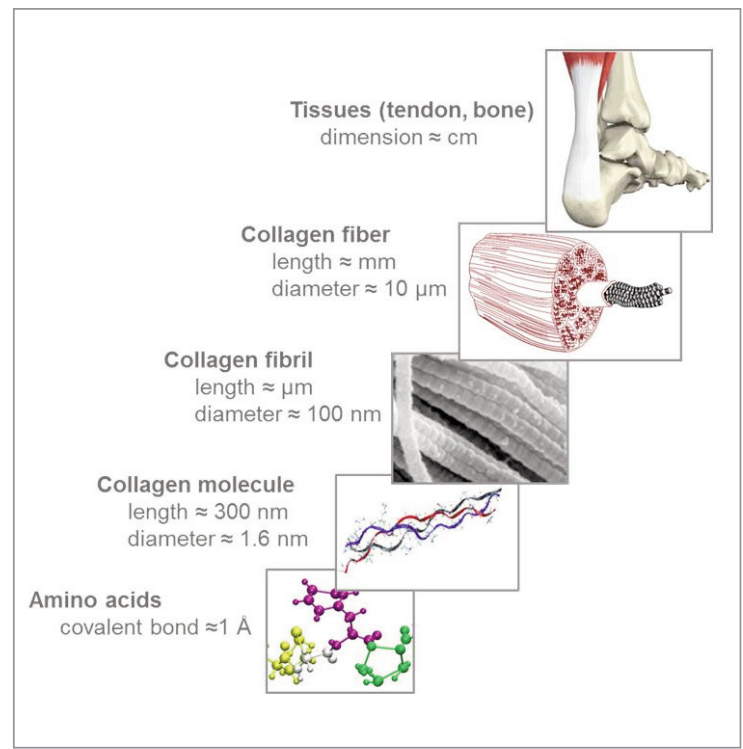

Figure 1. Hierarchical structure of collagen protein materials. Each collagen molecule is made of three peptide chains that form the $\approx 300 \mathrm{~nm}$ long triple helical collagen molecule. Collections of collagen molecules aggregate both in lateral and longitudinal directions to form fibrils. Fibrils in cornea are normally thin $(\approx 30 \mathrm{~nm})$ and uniform in diameter, while tissues such as tendon contain a wide-ranging distribution of diameters (100-500 nm). Fibrils include tiny hydroxyapatite crystals in bone tissue, which provide stiffness and compressive load resistance. In tendons and ligaments multiple fibrils make up collagen fibre, formed with the aid of proteoglycans. 


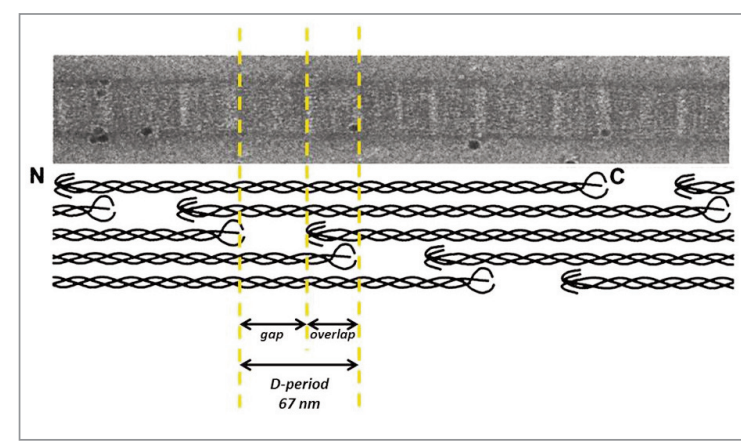

Figure 2. D-period of collagen fibrils. Electron microscope of a collagen fibril (top panel) and schematic representation of the axial arrangement of molecules in the D-periodic fibril (bottom panel).

er, where the molecular axes are also parallel to the fibril direction. This structure creates an observable periodicity known as the $D$-period whose length is $D=67 \mathrm{~nm}$, in which two region can be observed, the gap and overlap regions, emerging due to the fact that the length collagen molecule is not a multiple of the $D$-perioditself (Fig. 2). A particularly important feature for the mechanical properties of fibrils is the presence of enzyme-induced covalent crosslinking between molecules, which develops only with maturation of the tissues?.

At the next level of the hierarchy, the organization of the collagen structure varies greatly depending on the tissue. In tendons and ligaments, multiple fibrils make up the collagen fiber, which are formed with the aid of cross-linking macromolecules such as proteoglycans. In bone, the organic collagen protein matrix alone is not sufficient to provide the stiffness and resistance to compression required for this tissue (which has to carry considerable loads) and additional stiffening is provided by the inclusion of mineral hydroxyapatite crystals into collagen fibrils and particularly in the gap region, 8 . Skin and artery walls need to be flexible but arteries also need to resist blood pressure. This implies yet a different arrangement of collagen fibrils in combination with elastomeric molecules, such as elastin.

Due to the biological importance of collagen-based tissues there have been a wealth of investigation concerning the relationship between structure and properties in these complex materials, starting from early works (dated in the 50s) on of the structure of collagen molecules ${ }^{4}$. However, there are still open questions (e.g., concerning the mechanism of load transfer between the hierarchical scale of bone and tendons, or the onset of collagen-related diseases). A very promising technique to complement experiments in addressing these questions is represented by multi-scale modeling. In the following of this Chapter, successful application of atomistic and coarse-grain modeling techniques for the study of collagen materials are presented.

\section{Visco-elastic properties of collagen molecules}

In vertebrates, locomotion and movements are achieved through the generation of muscular forces that are then transmitted to joints. The force transmission (mechan- otransduction), which involves the storage, release and dissipation of energy, is provided by connective tissues such as ligaments and tendons ${ }^{10,11}$. Thus, in these tissues viscoelasticity is a fundamentally important feature, as both viscous (time-dependent) and elastic (time independent) contributions determine how ligaments and tendons accomplish their function as mechanotransducers. The mechanical behavior of these connective tissues is directly related to their complex hierarchical structure and to their specific macromolecular components ${ }^{12}$. The lowest hierarchical scale of several load-bearing tissues is represented by collagen type I molecules ${ }^{13}, 14$.

At the macro-scale (i.e., whole tissues and collagen fibers), the mechanical investigation of collagen-based tissues has been performed for several decades. As a consequence, it is well known that collagen-rich tissue present viscoelastic behavior, as established from a larger number of creep and relaxation tests that have been performed on both tendons and ligaments ${ }^{15-17}$. Based on experimental data, and starting from the pioneering work of Fung ${ }^{18}$, several constitutive and structural models have been developed to model the viscoelastic behavior of collagen at the tissues scale ${ }^{19-24}$.

On the other hand, fewer studies focused on the viscoelastic properties at smaller scales. At the fibril level, earlier investigations have been performed through small angle X-ray scattering 25 to assess the fibril deformation inside tissue specimens. However, in this set-up the load is not directly applied to single fibril and hence the response is averaged over several fibrils. Direct experimental studies of single fibrils are relatively recent and made possible due to the use of atomic force microscopy or micro-mechanical systems which allowed the study of the elastic ${ }^{26-30}$ and viscoelastic properties of collagen fibrils ${ }^{31,32}$.

Ultimately, at the molecular level several studies have been performed using both experimental and computational procedures; however most of these investigations focused solely on the elastic properties ${ }^{33-42}$. For this reasons, despite the abundant investigation of the viscoelastic behavior at the macro-scale and the very recent works on collagen fibril viscoelasticity, relatively little is known about the molecular and fibrillar origin of the time-dependent properties of collagenous tissues. It has been speculated that the elastic behavior of these tissues is due to the stretching of cross-linked collagen molecules and thus fibrils, whereas the energy dissipation (the viscous behavior) is thought to involve sliding of molecules and fibrils by each other during the tissue deformation ${ }^{12,} 43$. However, there is still no clear understanding of the mechanisms behind the viscoelastic behavior of collagenous tissue, and on the role of each hierarchical scale in determining the overall mechanical properties. An important outstanding question is whether or not molecular-level load relaxation effects are important for the viscoelastic properties measured at larger scales.

An approach to investigate the molecular origin of collagen viscoelasticity, is the use of in silico tests to study collagen molecules from the level of amino acids upwards. In this way it is possible to obtain quantitative data on the timedependent mechanical behavior of single collagen mole- 


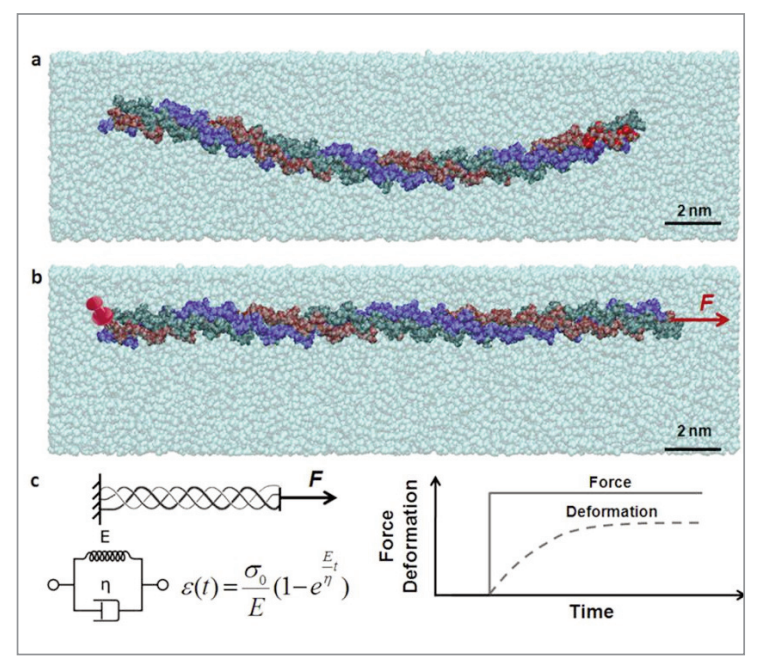

Figure 3. Snapshots of the collagen peptide in water box. Panel a shows the conformation of the full atomistic model of a collagen peptide solvated in water box and equilibrated for $30 \mathrm{~ns}$. After equilibration the molecule is subjected to virtual creep tests: one end of the collagen peptide is held fixed, whereas the other end is pulled with constant force (from $300 \mathrm{pN}$ to $3,000 \mathrm{pN}$ ) until end-to-end distance reaches equilibrium (Panel b). Panel c shows a schematic of the creep test; a constant force is applied instantaneously to the molecule and its response (deformation over time) is monitored. The mechanical response of collagen molecule is modeled using a KV model, from which molecular Young's moduls (E) and viscosity $(\eta)$ are calculated.

cules in order to assess molecular-level viscoelastic properties, and to scale up properties from there to larger tissue levels. In this approach, Steered Molecular Dynamics simulations are used to perform in silico creep tests (i.e., constant load tests) of collagen molecules, fitting the deformation response over time with a Kelvin-Voigt (KV) model. The KV model is a simple constitutive model consisting of a spring and a dashpot in parallel (Fig. 3), where this approach allows us to compute the Young's modulus, the viscosity and the characteristic relaxation time of a single collagen molecule.

In order to perform constant force SMD simulations, the three $\mathrm{N}$-terminal $\mathrm{C}_{\alpha}$ atoms are kept fixed whereas the three $\mathrm{C}$-terminal $\mathrm{C}_{a}$ atoms are subject to an instantaneous constant force. This set-up mimics a single molecule creep test, in which an instantaneous load is applied and the strain response in time is observed. The creep test simulations are run until asymptotic deformation and then strain-time curves obtained for collagen molecules during SMD simulations are fitted with the Kelvin-Voigt equation in order to estimate $E$ (Young's modulus) and $\eta$ (viscosity). The instantaneous stress $\sigma_{0}$ is calculated dividing the applied force by the crosssectional area of collagen, assuming a round cross-section and a radius of $0.55 \mathrm{~nm}$. The value of the radius is determined from dry collagenous tissue in which the lateral distance between molecules is found to be $1.1 \mathrm{~nm}^{44}$.

When subjected to creep tests, the engineering strain of the molecule shows an exponential increase until as-

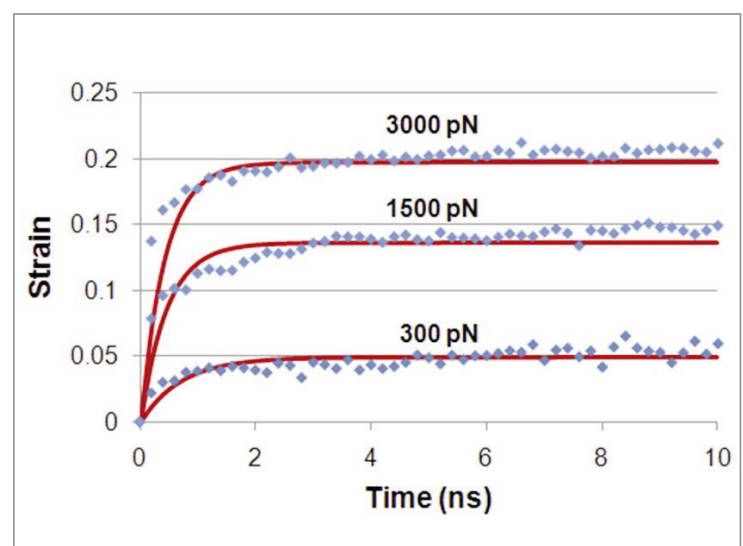

Figure 4. Single molecule creep test. Mechanical response of solvated collagen molecule to creep tests for three cases with increasing value of external force. Dots represent the experimental data, whereas curves represent the fitted curves using a Kelvin-Voigt model.

ymptotic value is reached (Fig. 4). This is the behavior typical of simple viscoelastic materials that can be characterized by a Young's modulus $E$, responsible for the elastic response, and a viscosity $\eta$, responsible for the viscous behavior. These results show that collagen molecule presents a non-linear elastic behavior, since the Young's modulus depends on the external load. In particular, for the loading conditions considered here $E$ ranges from 6 to $16 \mathrm{GPa}$, a finding that matches several experimenta| ${ }^{33}$, 34, 36, 37 and modeling studies 41, 45-47.

The collagen molecule viscosity has an average value of 3.84. $\pm 0.38 \mathrm{~Pa} \cdot \mathrm{s}$ and it is not significantly affected by the external load. Based on the elastic modulus and the viscosity, the characteristic relaxation time (defined, for a KV model, as $\eta / E$ ) is calculated, obtaining values in the range 0.24-0.64 ns and 0.13-0.27 ns for solvated and dry molecule, respectively. Although the elastic behavior has been previously investigated, no experimental data is available for the viscous component of a single collagen molecule. This could be attributed to the very fast relaxation time (on the order of nanoseconds) of single molecules, which hinders its assessment using available experimental techniques. However, a recent work ${ }^{48}$ demonstrated that Atomic Force Microscopy (AFM) can be successfully used to assess the viscoelastic properties of a single polypeptide chain, in this case polystyrene. Within this technique, referred to as "nanofishing" by the authors of that study, an AFM cantilever is mechanically oscillated at its resonant frequency during the stretching process. This enables the estimation of stiffness and viscosity of a single polymer chain with the use of a phenomenological model (the authors used a KV model). The polystyrene viscosity per unit length reported by the authors is $2.6 \cdot 10-9 \mathrm{Kg} / \mathrm{s}$, which agrees well with the findings for collagen molecules, i.e., $\approx 40 \cdot 10-9 \mathrm{Kg} / \mathrm{s}$ (considering a peptide length of $20 \mathrm{~nm}$ ). The slightly higher viscosity could be attributed to the fact that the polystyrene chain is a single strand, while the collagen peptide is in a triple helical configuration.

It is of great interest to discuss whether the two elements 


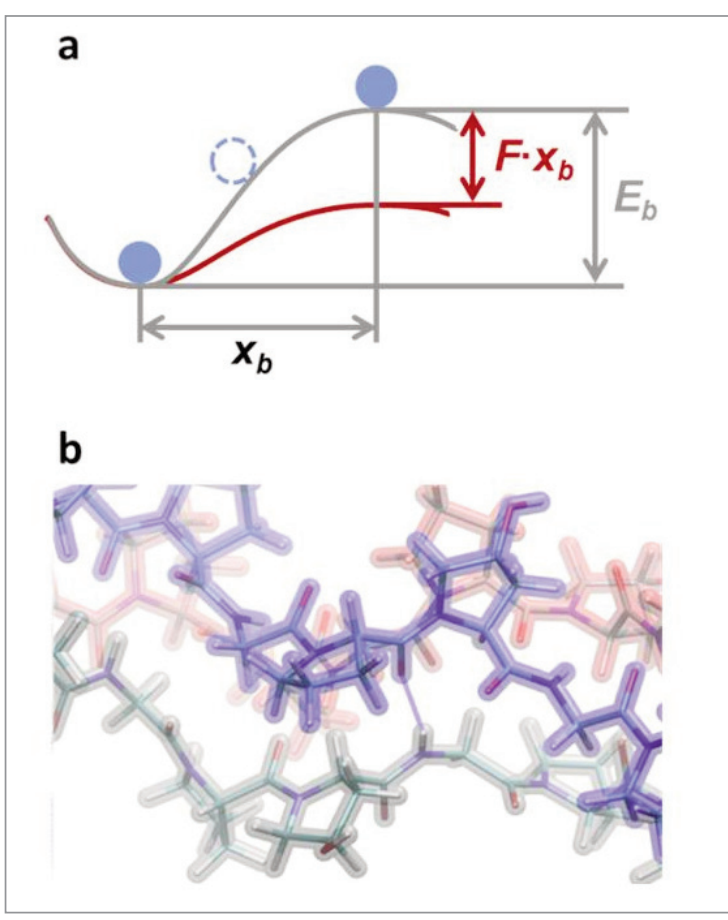

Figure 5. Statistical theory to predict bond rupture mechanics. The graph depicts the energy as a function of a deformation variable (a), along a particular pathway that leads to bond rupture, where $E_{b}$ is the energy barrier corresponding to the transition state. Panel b shows an interchain $\mathrm{H}$ bond in collagen.

of the $\mathrm{KV}$ model, i.e. the purely elastic spring and the purely viscous dashpot, have an actual physical meaning. A likely explanation would be that the elastic spring corresponds to the protein backbone, while the damping effect could be attributed to the interchain $\mathrm{H}$-bonds. The backbone deformation include dihedral, angle and bond deformation, which are terms expressed by harmonic (or similar) functions in the molecular dynamics force field, and thus result in an elastic response to stretching. On the other hand, the viscous behavior may be due to the breaking and reforming of $\mathrm{H}$-bonds, in particular $\mathrm{H}$-bonds between the three collagen chains. Statistical mechanics based theories, and in particular Bell's model ${ }^{49}$, has been applied to study the breaking of $\mathrm{H}$-bonds (see, e.g. reference ${ }^{50}$ Fig. 5). It has been shown that the velocity at which a $\mathrm{H}$-bond breaks is a function of the external force:

$v=\frac{x_{b}}{\tau_{0}} e^{-\left[\frac{E_{b}-F \cdot x_{b} \cos \theta}{k_{b} T}\right]}$

where $v$ is the velocity at which the $\mathrm{H}$-bond breaks, $x_{b}$ is the distance between the equilibrium state and the transition state, $\tau_{0}$ is the reciprocal of the bond natural frequency, $E_{b}$ is the bond energy, $F$ is the applied force, $\theta$ is the angle between the direction of the reaction pathway of bond breaking and the direction of applied load $F, k_{b}$ is the Boltzmann constant and $T$ is the temperature. The equation above shows that the mechanical response of the $\mathrm{H}$-bonds is time-dependent, matching the behavior of the dashpot in the KV model very well (for which the stress is a function of the loading rate). Therefore the timedependent rupture of $\mathrm{H}$-bonds are likely responsible for the dissipative behavior observed in collagen molecule mechanics.

\section{Atomistic modeling of collagen fibrils}

In order to complement experimental approaches, molecular modeling provides a powerful approach to describe the molecular mechanics of collagen. Earlier studies were based on short collagen-like peptides obtained from $x$-ray crystallography ${ }^{51-54}$. These early molecular simulation studies used these short collagen molecules $38,39,41,46,55$ 61 that were typically limited to less than $10 \mathrm{~nm}$ length or more than a factor of 30 smaller than actual molecules found in collagen tissues. The resulting elastic modulus of these short collagen peptides was found to be on the order of $4 \mathrm{GPa}$, and thus much greater than the typical Young's moduli measured for macro-scale collagen tissues but in agreement with single molecule studies ${ }^{33-36,62}$. In order to determine how collagen-based structures confer mechanical properties to tissues like skin, tendon and bone, and to identify how cells interact with the ECM, the understanding of the mechanics at different hierarchical levels and their interplay from a biochemical and molecular level upwards is essential. Earlier work has demonstrated that mechanical strain is distributed over distinct hierarchical levels (molecules, fibrils, fibres) ${ }^{63-65}$ and that collagen tissue stretching involves concurrent deformation mechanisms. The significance of defining the material properties of collagenous tissues from the biochemistry level upwards is evident when considering the effect of mutations in collagen, which can result in incorrectly folded collagen protein that cause a variety of severe and sometimes deadly pathologies, such as Ehlers-Danlos syndrome or osteogenesis imperfecta (brittle bone disease) ${ }^{66}$. Early collagen microfibril and fibril models represent the supramolecular arrangement in collagenous tissues in a simplified way, using a two-dimensional lattice of mesoscopic beads ${ }^{67,68}$ or extremely short collagen peptides ${ }^{38}$, $39,41,46,55-60$. These models do not account for the biochemical details and are much smaller than the typical length-scales of collagen molecules found in collagen microfibrils and fibrils. The reason for these approximations was the lack of crystallographic details of the collagen molecule, which have been obtained only for short collagenlike peptides with lengths below $\approx 10 \mathrm{~nm}^{51-23}$, until the work of Orgel and coworkers, that obtained the in situ structure of full length collagen type I molecule ${ }^{69}$ (Protein Data Bank identification code 3HR2) (Fig. 6). This structure, obtained by employing conventional crystallographic techniques in $\mathrm{x}$-ray fiber diffraction experiments, resolved for the first time the specific three-dimensional arrangement of collagen molecules in naturally occurring fibrils, including the $\mathrm{N}$ - and $\mathrm{C}$-telopeptides.

Starting from the structure reported in ${ }^{69}$, which includes only backbone alpha carbons and the primary sequence of rattus norvegicus, Gautieri et al. ${ }^{70}$ used homology modeling to obtain a full-atom structure with the human collagen sequence (Fig. 7). The molecular packing topolo- 


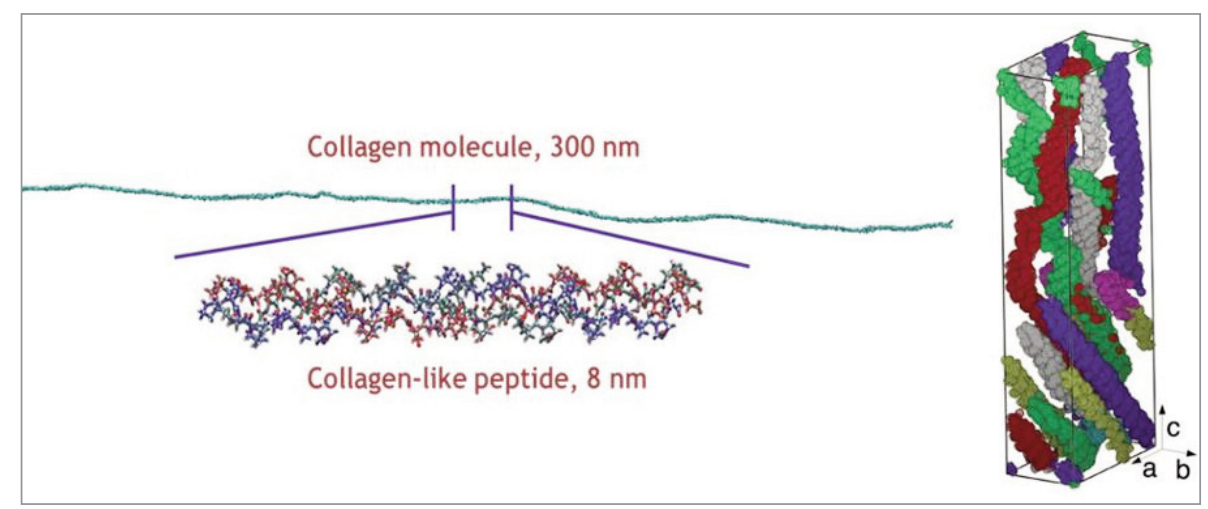

Figure 6. Comparison of collagen-like peptides with the actual collagen molecule (left) and unit cell of the low-resolution X-ray crystallography of the collagen microfibil described in (69) (right).

gy obtained by the periodic repetition of the unit cell lead to quasi-hexagonally packed collagen molecules which interdigitates with neighboring molecules to form a super twisted right-handed microfibril and to the well-known $D$ banding periodicity seen in AFM images of collagen fibrils. The fibril model is solvated using the solvate plug in of GROMACS by adding SPC water molecules. Since the molecule at physiological $\mathrm{pH}$ includes a net charge (positive net charge +34$)$, counterions $\left(\mathrm{Cl}^{-}\right)$are added in order to keep the system neutral. The fibril model is equilibrated through several ns molecular dynamics simulations at a temperature of $310 \mathrm{~K}\left(37^{\circ} \mathrm{C}\right)$, and with 1 bar pressure to ensures structural convergence. In order to assess the mechanical properties of the atomistic models, molecular dynamics simulations with increasing constant mechanical stress in tension along the fibril axis has been perfor med, while maintaining the pressure on the other axes constant at 1 bar. The applied stresses are in the range from 0 to $200 \mathrm{MPa}$, applied during $20 \mathrm{~ns}$ molecular dynamics simulation for each load applied.

The equilibrated collagen microfibril (Fig. 8) has a density of $\approx 1.19 \mathrm{~g} / \mathrm{cm}^{3}$, a value that is halfway between the den- sity of water and the density of dehydrated collagen, which has been estimated at $1.34 \mathrm{~g} / \mathrm{cm}^{3} 71$. A Ramachandran analysis of the solvated system shows that the collagen microfibrils in a region of the diagram (Psi $\approx 150^{\circ}$, Phi $\approx-75^{\circ}$ ) that is characteristic of the polyproline II chain and thus of collagen-like peptides, in good agreement with earlier experimental structural studies ${ }^{72}$.

When subjected to mechanical load, collagen microfibrils feature two distinct deformation regimes. In the small-strain regime $(<10 \%)$ the predicted Young's modulus is $\approx 300$ $\mathrm{MPa}$, while in the large-strain regime $(>10 \%)$ the microfibril shows a severely increased tangent stiffness, with a Young's modulus of $\approx 1.2 \mathrm{GPa}$ (Fig. 8 ). The results of nanomechanical testing of collagen microfibrilare in agreement with experimental results obtained for the small strain regime based on several techniques such as x-ray diffraction ${ }^{25}$, Atomic Force Microscopy (AFM) ${ }^{73}, 74$ and the use of micro-electro-mechanical systems $(\mathrm{MEMS})^{28,29,75}$. For the larger-strain regime there exists less experimental information and available data tend to be more scattered. For example, Shen et al. ${ }^{28}$ showed a relatively large variability of collagen fibril behaviors at large

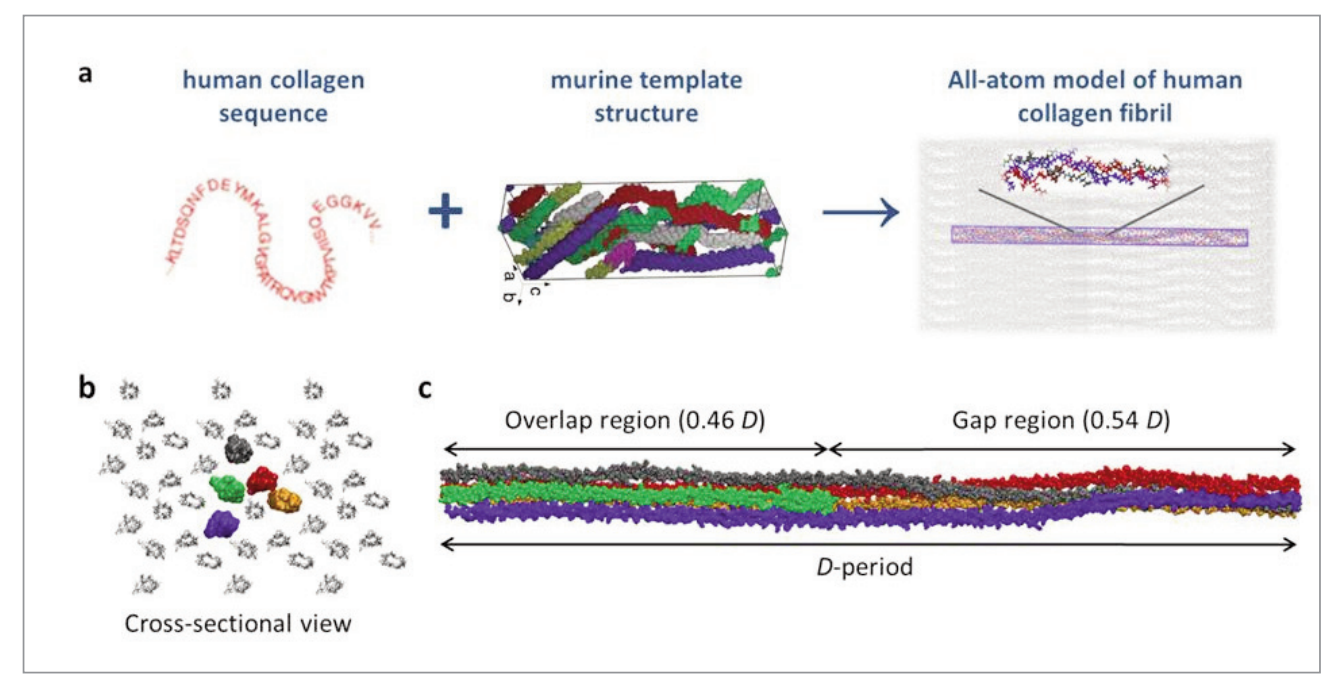

Figure 7. Schematic of the homology modeling process, in which a target sequence is applied to a template structure. The use of the murine template structure is possible thanks to the high sequence homology between human and murine collagen type I (a). Panel b shows the quasi-hexagonal cross-section of the microfibril model and panel c the D-period divided into gap and overlap regions. 


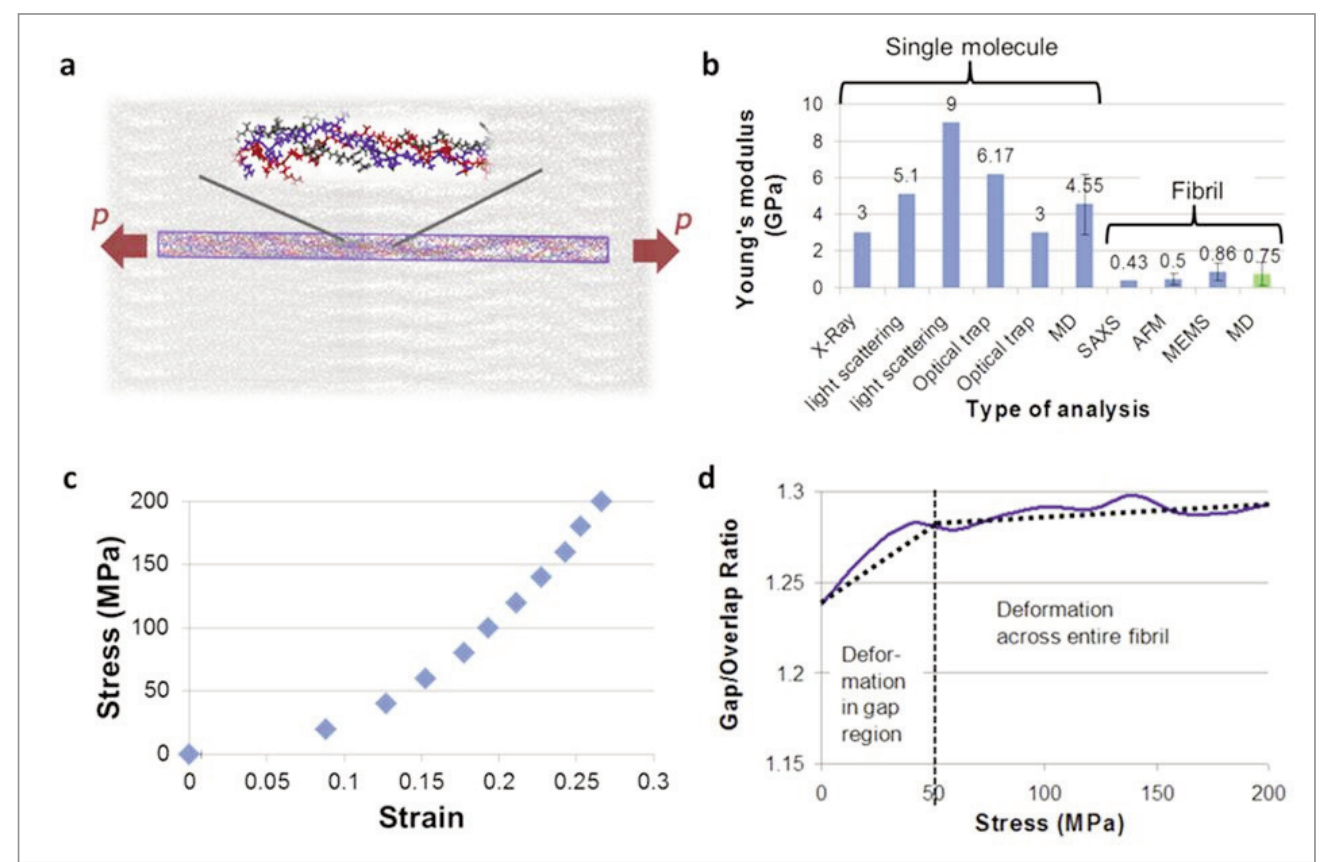

Figure 8. Schematic of the in silico mechanical loading (a) and comparison of modeling results with experimental results at fibril and molecular scale (b). Stress-strain relationship for collagen type I microfibril (c) and analysis of the gap/overlap ratio, showing that for small deformation the gap region is deforming more (d).

deformation, which suggested either strain-hardening or strain-softening depending on the fibril investigated. A direct comparison of the mechanical properties of single collagen molecules versus that of collagen microfibrils suggests that the mechanical properties are strongly scale dependent (Fig 8).

The atomistic model enables to observe atomistic and molecular deformation mechanisms not directly accessible to experimental techniques, and thereby to explain the molecular origin of mechanical properties at different hierarchical levels and levels of strain. It is observed that for small deformation the collagen molecules' end-to-end distance increases linearly until the microfibril strain reaches $10 \%$ (corresponding to $\approx 50 \mathrm{MPa}$ stress), the strain at which the microfibril stiffness increases drastically. Beyond this point the molecular end-to-end still continues to increase but the slope of curve is significantly lower. This can be explained due to the fact that below $10 \%$ strain the collagen molecule is straightened within the microfibril and thereby loses its kinked arrangement, while beyond the $10 \%$ strain mark the molecule itself is being stretched, resulting in a larger mechanical resistance to deformation. The increase in the gap to overlap ratio in the small-strain regime (Fig. 8) suggests that the initial straightening is concentrated in the gap regions where the molecular packing density is lower and molecules are less organized, with more kinks. This model thereby confirms suggestions made by Fratzl et al. ${ }^{2,3,76}$. Once the entire capacity to molecular straightening is exhausted and all collagen molecules assume a straight configuration oriented in the direction of the pulling axis, collagen molecules themselves undergo stretching, leading to significantly increased tangent microfibril stiffness, at strains in excess of $10 \%$. The combination of these two mechanisms, molecular straightening and molecular stretching, effectively lead to an increase of the $D$-period, in agreement with experimental results ${ }^{77}$. Other mechanisms, such as molecular sliding may take place at larger strains in excess of $30 \%$ as described in earlier studies of the deformation mechanisms ${ }^{25,76}$.

\section{Coarse-grain modeling of collagen}

As seen in the previous paragraph, the study of collagen fibrils and fibers with full atomistic simulations is currently challenging, which represents an important limitation since this scale in the structural hierarchy of collagen is most relevant for physiological function. Other limitations exist with respect to the accessible time-scale, where most full-atomistic simulations are limited by a few hundred nanoseconds. However, many relevant materials phenomena such as tissue deformation and failure (e.g. under disease conditions) emerge at much longer timescales.

A promising strategy to overcome these limitations is to decrease the number of degrees of freedom by grouping atoms into pseudo-atoms (or particles) referred to as beads. This represents the basis of the so-called coarsegrained approach ${ }^{78,79}$, where starting at the nanoscale, it is possible to derive parameters for higher hierarchical levels, up to the macroscale by systematically feeding information from smaller, more accurate to larger, more coarse levels ${ }^{80,81}$. The development of a coarse-grained model provides a powerful path towards reliable modeling of full-length collagen molecules and its higher level hierarchical structures. Specifically, coarse-grained mod- 
els allow the study of more complex systems, up to micrometer dimension and millisecond duration ${ }^{82}$. Earlier work of coarse-graining collagen molecules grouped hundreds of atoms into particles ${ }^{38,67}$. This level of coarsegraining, however, is at a relatively coarse level where information about biochemical features (e.g. amino acid sequence) cannot be represented directly. However, the incorporation of biochemical features is crucial in a computational materiomics approach where material properties are elucidated at multiple scales.

Several other coarse-grained models suitable for proteins have been developed and successfully applied ${ }^{83}$. Particularly, the Martini coarse-grained model ${ }^{84}$, developed by Marrink and coworkers, was initially applied to membrane lipids, later extended to proteins ${ }^{85}$ and to carbohydrates $^{86}$. It has been successfully applied to gain insights about different biological molecules such as membrane proteins $^{87,88}$, ion channels ${ }^{89}$ and liposomes ${ }^{90}$.

The Martini model provides a suitable level of coarse-graining as it retains information about the chemistry specific to the amino acid sequence (as side chains are modeled depending on the type of the amino acids). All amino acids in the Martini force field are modeled with a number of beads that varies depending on the steric volume of each amino acid ${ }^{85}$. The general mapping rule is that four heavy atoms (that is, non-hydrogen atoms) are grouped together into one bead. Further, one bead describes the backbone, while others are added to represent the side chain. The number of beads used to model a specific residue therefore varies depending on the dimensions of the side chain of the amino acid. Small amino acids such as glycine or alanine are described by just one bead, while larger amino acids, like phenylalanine, tyrosine and tryptophan are modeled with up to five beads. The model also takes in account the polarity of every bead, described by a letter (P: polar; C: apolar; N: nonpolar; Q: charged) and a number (from 1: low polarity to 5: high polarity). Further, a letter is used to characterize a residue's hydrogen bonding capability (d: donor; a: acceptor; da: donor and acceptor; 0: none). These bead types, which correspond to atom types in the atomistic modeling framework, are used to describe the non-bonded interactions between beads. For each pair of bead types, a set of parameters for electrostatic and van der Waals potentials is defined. The best choice of particle types for amino acids was obtained by the authors of the original Martini force field for proteins by comparing simulation results and experimental measurements of water/oil partitioning coefficients of the amino acid side-chain analogues ${ }^{85}$.

However, although the Martini force field is suitable for a wide number of applications and its validity has been proven through several simulations and comparisons both with atomistic and with experimental data, it cannot be applied to model collagen molecules in the original formulation. This is because of two reasons. First, the existing formulations of Martini lack parameters for hydroxyproline (a non-standard amino acid, found solely in collagen and formed through post-translational modification of proline). Second, it lacks parameters to describe the triple helical configuration of a collagen molecule. Thus, recent efforts have been addressed to overcome these limitations and addresses two major points.

In the Martini model, proline is modeled through the use of two beads, one for the backbone (bead type C5) and one for the side-chain (bead type AC2) as shown in Figure 9. Hydroxyproline derives directly from proline via the addition of a hydroxyl group on its side-chain. Therefore, hydroxyproline is also modeled using two beads in the extended MARTINI model. For the backbone bead is represented with the same bead type as for proline. The side chain, however, due to the presence of the hydroxyl group, shows a higher polarity level than proline. This aspect was already demonstrated in the work of Black and Mould ${ }^{91}$, who calculated an index that takes into account the hydrophobicity of all amino acid side-chains, including hydroxyproline. Matching the bead types assigned by Marrink and the hydrophobicity values derived from Black and Mould, the hydroxyproline side-chain bead polarity, in the Martini notation, is determined. This parameter ranges from 0 to 1 , where 1 is the most hydrophobic amino acid (phenylalanine), while 0 is the most hydrophilic one (arginine). The value reported for hydroxyproline is 0.527 , which is found between threonine $(0.450)$ and cysteine $(0.680)$ values. In the Martini force field, threonine side chain is modeled as a P1 bead, while cysteine as a C5 bead. Considering this result, hydroxyproline is assigned a P1 value for the hydroxyproline side-chain, since its hydrophobicity value is closer to the value for threonine. The Martini model also takes into account the secondary structure of a protein, such as alpha-helix, beta-sheet, extended turns, or bends. The characteristic triple helical structure of collagen molecules cannot be described by any of the secondary structure parameters included in original Martini model. Thus, the bond distances, angles and dihedral parameters specific to the collagen triple helix have been assessed. The Martini bond potential forms are maintained, which are described as follows:

$$
\begin{aligned}
& V_{b}=\frac{1}{2} K_{b}\left(d_{j j}-d_{b}\right)^{2}, \\
& V_{a}=\frac{1}{2} K_{a}\left[\cos \left(\varphi_{i k}\right)-\cos \left(\varphi_{a}\right)\right]^{2} \\
& V_{d}=K_{d}\left[1+\cos \left(n \psi_{j u}-\psi_{d}\right)\right]
\end{aligned}
$$

Where $V_{b}, V_{a}$ and $V_{d}$ are the potential energy terms of bond stretching, angle deformation and dihedral deformations, respectively. Bonded interactions act between bonded sites $i, j, k, I$ with a distance at the equilibrium of $d_{b}$, angle $\phi_{a}$ and dihedral angles $\psi_{d}$ and with elastic stiffness of $K_{b}, K_{a}$ and $K_{d}$ (for bond, angle and dihedral, respectively).

The triple helical geometrical parameters of the collagen triple helix (that is, $d_{b}, \phi_{a}$ and $\psi_{d}$ ) are obtained by performing a statistical analysis on a set of five collagen-like molecules available in the Protein Data Bank ${ }^{92}$ (identification codes: 2DRT, 1K6F, 1QSU, 1CAG, 1V6Q). The bond lengths between backbone beads, bonding angles and dihedral angles are computed on the basis of these five crystallographic structures, providing a bond reference length $\left(d_{b}\right)$ of $0.365 \pm 0.07 \mathrm{~nm}$, a bonding reference angle $\left(\phi_{a}\right)$ of $119.2 \pm 8.72^{\circ}$, and a dihedral reference angle $\left(\psi_{d}\right)$ of $-89.3 \pm 9.76^{\circ}$. Figure 9 shows the details of a coarsegrained model of a collagen chain with the angular geometrical parameters. 


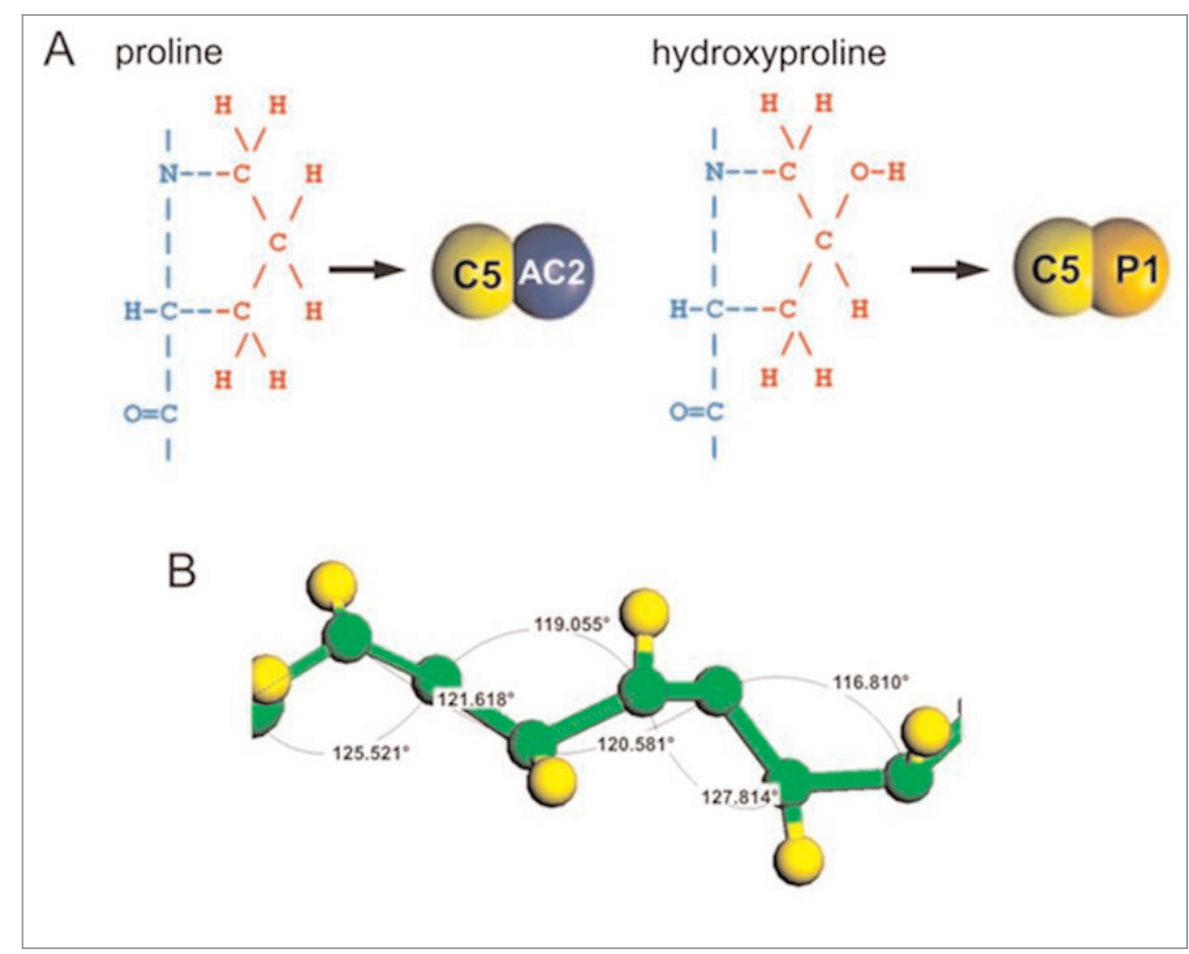

Figure 9. Parameterization of the force field of the MARTINI force field including the hydroxyproline residue (Panel A) and geometrical features (angles) of the coarse-grained collagen triple helix (Panel B).Hydroxyproline directly derives from proline adding a hydroxil group to its side chain, giving rise to an increased polarity level. Atomistic structures of both proline and hydroxyproline are shown on the left side of Panel A, while the bead representation using MARTINI model notation is shown on the right side of Panel A. Panel B shows the analysis of the angle between backbone beads of collagen-like peptides. The statistical analysis is performed on five collagen PDB entry (2DRT, 1K6F, 1QSU, 1CAG, 1V6Q); these collagenlike peptides are coarse-grained and the bond lengths, angles and dihedrals between backbone beads are analyzed.

In order to assess the stiffnesses of bond, angle and dihedrals (respectively, $K_{b}, K_{a}$ and $K_{d}$ ), simple atomistic oligopeptides are considered. In order to derive the bonding constant $K_{b}$, a glycine-proline structure is chosen. In order to derive the angle elastic constant $K_{a}$, a glycine-proline-hydroxyproline oligopeptide is considered. Finally, to obtain the dihedral elastic constants $K_{d}$ a glycine-prolinehydroxyproline-glycine molecule is studied. These structures are considered both in their atomistic form and in their coarse-grained form, and the parameters of the coarse-grain model (i.e, $K_{b}, K_{a}$ and $K_{d}$ ) are identified matching force-extension curves obtained from atomistic and coarse-grained steered molecular dynamics simulations. For the coarsegrained models, the spring constants (that is, the parameters $K_{b}, K_{a}$ and $K_{d}$ ) are initially set equal to the values adopted in the original Martini force field for an alpha helix secondary structure ${ }^{85}$ and then gradually changed in order to best fit the atomistic force-extension curves so that they show the same stiffnesses. The values that best replicate the atomistic behavior are $K_{b}=1250 \mathrm{kJmol}^{-1} \mathrm{~nm}^{-2}, K_{a}=150$ $\mathrm{kJmol}^{-1}$ and $K_{d}=100 \mathrm{kJmol}^{-1}$.

In order to validate the extended MARTINI force field model, an $8 \mathrm{~nm}$ long collagen-like molecule (similar to the PDB entry used to assess the geometrical features) is pulled under the same conditions previously described. The chosen molecule is [(glycine-proline-hydroxyproline) $\left.)_{10}\right]_{3}$, which represents an "ideal" reference collagen molecule that was used in several earlier molecular dynamics works ${ }^{38,93}$. The equilibrated coarse-grained collagen peptide is pulled using a deformation rate of $1 \mathrm{~m} / \mathrm{s}$ and the Young's modulus of the coarse-grain collagen molecule is then calculated from the slope of the force-deformation plot. Assuming a cylindrical geometry, and considering a collagen molecular radius of $0.8 \mathrm{~nm}$, the Young's modulus is obtained equal to $4.62 \pm 0.41 \mathrm{GPa}$.

The whole structure of the heterotrimeric type I collagen molecule is investigated in order to derive the persistence length. The full length coarse-grained tropocollagen model is divided into five $60 \mathrm{~nm}$ peptide strands to reduce the computational cost. Then, the flexibility of the structures is analyzed from molecular dynamics trajectories of 350 ns each, obtaining the persistence length through the following expression ${ }^{94}$ :

$\log \left\langle\cos \theta>=-\frac{s}{L_{p}}\right.$

As shown in the inlay in Figure 10, the variable $\theta$ denotes the angle between two segments along the molecule separated by contour length $s$ (that is, the sum of the segment lengths), and $L_{p}$ is the persistence length, which provides information about a molecule's flexibility. In order to evaluate the persistence length, the molecule is divided into several smaller segments. Each segment (as shown in the inlay in Fig. 10), is delimited by the center of mass of the three 


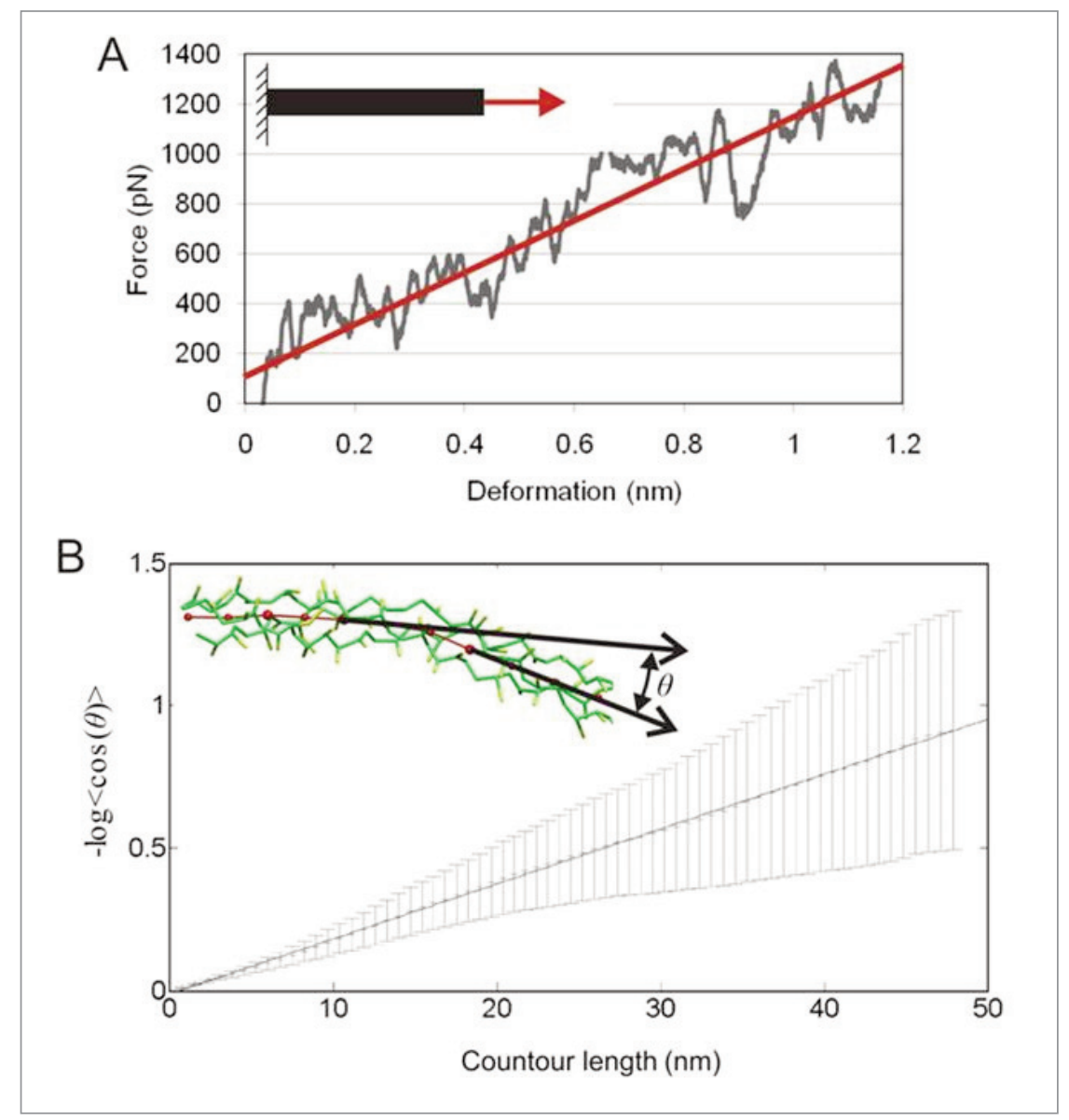

Figure 10. Mechanical analysis of collagen molecules. Panel A: Force-deformation plot up to $15 \%$ strain (1.2 $\mathrm{nm}$ ) for the pulling test of a [(glycine-proline-hydroxyproline) 10$]_{3}$ collagen-like peptide (straight line) and linear regression (dashed line) used to obtain the molecular elastic constant. Panel B: Plot of $-\log <\cos (\theta)>$ versus contour length calculated for the coarsegrain model of the first strand $(\approx 60 \mathrm{~nm})$ of human collagen type I molecule. The linear regression of the data for the five different strands leads to an average persistence length of $\approx 51.5 \mathrm{~nm}$ for human type I collagen.

equivalent glycine residues belonging to the three different collagen chains. The plot of $-\log <\cos (\theta)>$ versus $s$ is obtained, where the average of $\cos (\theta)$ is calculated over the full molecular dynamics simulations trajectories. Figure 10 shows the plot of $-\log <\cos (\theta)>$ versus the contour length $s$. The value of the persistence length $L_{p}$ is then obtained from the inverse of the slope after a linear regression of all data, giving a value of $L_{p}=51.5 \pm .6 .7 \mathrm{~nm}$.

The persistence length found based on the coarse-grained model is close to the value obtained by Hofmann and coworkers $^{35}$, who found a value of $57 \pm 5 \mathrm{~nm}$ for collagen type I molecule through an electron microscopy analysis. On the other hand, a literature analysis shows that great variability in persistence length value can be found considering different kinds of experimental tests. The pioneering work in this analysis is represented by Utiyama and coworkers ${ }^{95}$, who considered sedimentation constants and the intrinsic viscosity of purified collagen molecules and calculated a value for the persistence length close to $130 \mathrm{~nm}$. Saito and coworkers ${ }^{96}$ considering the hydrodynamic properties of collagen derived the intrinsic viscosity and the sedimentation constant of collagen and, from these values, the persistence length, equal to 160$180 \mathrm{~nm}$. In another experiment, Nestler and coworkers ${ }^{97}$ measured the dynamic viscoelastic properties of dilute solutions of collagen molecules. From the obtained values of intrinsic viscosity and rotational relaxation time, they found a value for the persistence length of about $170 \mathrm{~nm}$. In more recent work, Sun and co-workers ${ }^{62}$ used optical tweezers in order to obtain, from force-extension plots fitted to the Marko-Siggia entropic elastic model ${ }^{98}$, the collagen persistence length, which was found to be $14.5 \pm 7.3$ $\mathrm{nm}$. Finally, molecular simulation studies were used to perform bending tests on a collagen-like peptide, predicting the bending stiffness of the collagen molecule and, from this result, evaluate the persistence length of collagen to be in the range of $16-24 \mathrm{~nm}^{99}$. The variability that can be found in literature for the collagen persistence length value depends on the used experimental setup, for example electron micrographs or optical tweezers, on the experimental conditions (how the sample is extracted and prepared), on the method applied and the parameters used to fit experimental data with theoretical models. Despite the large variability of experimental values, the val- 
ue obtained with the coarse grained model is in good agreement with the consideration of collagen as a semiflexible molecule with a rod like configuration.

Due to its contour length of $300 \mathrm{~nm}$, all-atom simulations with explicit solvent are prohibitive since they would require excessive computational resources due to the very large number of particles. The reduction by roughly a factor of 10 in the coarse-grained description provides a significant speed-up that facilitates the direct simulation of much longer molecules. Furthermore, the typical time step used in classical molecular dynamics simulations (1-2 fs) allows the modeling only on the nanoseconds time scale. Whereas the coarse-grained model enables to use much longer time-steps, on the order of $20-40$ fs. Considering the combined effect of the reduction of the number of particles and the increased time step, the coarsegrained approach leads to a total speed-up of 200-400 with respect to atomistic simulations.

With this significant computational speed-up, the coarsegrained modeling framework opens many possibilities for future studies, particularly at the scale of collagen fibrils and possibly fibers. However, the Martini coarse grain approach is only valid when the phenomena under study do not involve changes of the secondary structure. In the context of collagen, only events that do not involve unfolding of the collagen triple helix can be correctly modeled using the MARTINI force field formulation. While unfolding of molecules is likely to play an important role in large deformation and fracture of collagenous tissues, the mechanical stresses experienced by collagen tissues under small mechanical loads may not lead to unfolding at the molecular level. This is because strain is distributed over several hierarchical levels (i.e., fibers, fibrils, molecules) and involves several concurrent mechanisms (fiber uncrimping, proteoglycan-mediated fibril sliding, molecular slippage and molecular elongation).

\section{References}

1. Chamberlain $P$, Drewello $R$, Korn $L$, et al. Construction of the Khoja Zaynuddin mosque: use of animal glue modified with urine. Archaeometry 2011; 53:830-841.

2. Fratzl P, Weinkamer R. Nature's hierarchical materials. Progress in Material Science 2007; 52:1263-1334.

3. Fratzl P. Collagen: Structure and Mechanics. New York; Springer 2008.

4. Ramachandran GN, Kartha, G. Structure of collagen. Nature $1955 ; 176: 593-595$.

5. Rich A, Crick FHC. The structure of collagen. Nature 1955; 176:915-916.

6. Petruska JA, Hodge AJ. Subunit Model for Tropocollagen Macromolecule. Proceedings of the National Academy of Sciences of the United States of America 1964; 51:871-876.

7. Eyre DR, Weis MA, Wu JJ. Advances in collagen cross-link analysis. Methods 2008; 45: 65-74.

8. Currey JD. Bones: Structure and Mechanics. Princeton, NJ; Princeton University Press 2002.

9. Weiner S, Wagner HD. The material bone: Structure mechanical function relations. Annual Review Of Materials Science 1998; 28:271-298.

10. Alexander RM. Elastic Energy Stores in Running Vertebrates. American Zoologist 1984; 24:85-94.

11. Alexander RM. Animal Mechanics. Oxford, UK; Blackwell
Scientific 1983.

12. Silver FH, Freeman JW, Horvath I, Landis WJ. Molecular basis for elastic energy storage in mineralized tendon. Biomacromolecules 2001; 2:750-756.

13. Kadler KE, Baldock C, Bella J, Boot-Handford RP. Collagens at a glance. Journal of Cell Science 2007; 120:1955-1958.

14. Fratzl P. Collagen: Structure and Mechanics; Springer (New York) 2008.

15. Wang XT, Ker RF. Creep-Rupture of Wallaby Tail Tendons. Journal of Experimental Biology 1995; 198:831-845.

16. Rigby BJ, Hirai N, Spikes JD, Eyring H. The Mechanical Properties of Rat Tail Tendon. J Gen Physiol 1959; 43:265283.

17. Sasaki N, Shukunami N, Matsushima N, Izumi Y. Time-resolved X-ray diffraction from tendon collagen during creep using synchrotron radiation. Journal of Biomechanics 1999; 32:285-292.

18. Fung YC. Elasticity of soft tissues in simple elongation. Am J Physiol 1967; 213:1532-1544.

19. Haut RC, Little RW. A constitutive equation for collagen fibers. J Biomech 1972; 5:423-430.

20. Egan JM. A constitutive model for the mechanical behaviour of soft connective tissues. J Biomech 1987; 20:681-692.

21. Woo SLY, Johnson GA, Smith BA. Mathematical-Modeling of Ligaments and Tendons. Journal of Biomechanical Engineering-Transactions of the Asme 1993; 115:468-473.

22. Puxkandl R, Zizak I, Paris O, et al. Viscoelastic properties of collagen: synchrotron radiation investigations and structural model. Philosophical Transactions Of The Royal Society Of London Series B-Biological Sciences 2002; 357:191197.

23. Pena E, Pena JA, Doblare M. On modelling nonlinear viscoelastic effects in ligaments. Journal of Biomechanics 2008; 41:2659-2666.

24. Screen HRC, Anssari-Benam A, Bader DL. A combined experimental and modelling approach to aortic valve viscoelasticity in tensile deformation. Journal of Materials Science-Materials in Medicine 2011; 22:253-262.

25. Sasaki N, Odajima S. Elongation mechanism of collagen fibrils and force-strain relations of tendon at each level of structural hierarchy. Journal Of Biomechanics 1996; 29:11311136.

26. Van Der Rijt JAJ, Van Der Werf KO, Bennink ML, Dijkstra PJ, Feijen J. Micromechanical testing of individual collagen fibrils. Macromolecular Bioscience 2006; 6:699-702.

27. Svensson RB, Hassenkam T, Grant CA, Magnusson SP. Tensile Properties of Human Collagen Fibrils and Fascicles Are Insensitive to Environmental Salts. Biophysical Journal 2010; 99:4020-4027.

28. Shen ZL, Dodge MR, Kahn H, Ballarini R, Eppell SJ. Stressstrain experiments on individual collagen fibrils. Biophysical Journal 2008; 95:3956-3963.

29. Eppell SJ, Smith BN, Kahn H, Ballarini R. Nano measurements with micro-devices: mechanical properties of hydrated collagen fibrils. Journal Of The Royal Society Interface 2006; 3:117-121.

30. Shen ZLL, Dodge MR, Kahn H, Ballarini R, Eppell SJ. In Vitro Fracture Testing of Submicron Diameter Collagen Fibri Specimens. Biophysical Journal 2010; 99:1986-1995.

31. Shen ZL, Kahn H, Ballarini R, Eppell SJ. Viscoelastic properties of isolated collagen fibrils. Biophys J 2011; 100:30083015.

32. Svensson RB, Hassenkam T, Hansen P, Magnusson SP. Viscoelastic behavior of discrete human collagen fibrils. Journal of the Mechanical Behavior of Biomedical Materials 2010; 3:112-115.

33. Harley R, James D, Miller A, White JW. Phonons And Elastic-Moduli Of Collagen And Muscle. Nature 1977; 267:285-287. 
34. Cusack S, Miller A. Determination Of The Elastic-Constants Of Collagen By Brillouin Light-Scattering. Journal Of Molecular Biology 1979; 135:39-51.

35. Hofmann H, Voss T, Kuhn K, Engel J. Localization Of Flexible Sites In Thread-Like Molecules From Electron Micrographs Comparison Of Interstitial, Basement Membrane And Intima Collagens. Journal Of Molecular Biology 1984; 172:325-343.

36. Sasaki N, Odajima S. Stress-strain curve and Young's modulus of a collagen molecule as determined by the X-ray diffraction technique. Journal of Biomechanics 1996; 29:655658.

37. Sun YL, Luo ZP, Fertala A, An KN. Direct quantification of the flexibility of type I collagen monomer. Biochemical And Biophysical Research Communications 2002; 295:382-386.

38. Buehler MJ. Atomistic and continuum modeling of mechanical properties of collagen: Elasticity, fracture and selfassembly. J. Mater. Res. 2006; 21:1947-1961.

39. Gautieri A, Vesentini S, Montevecchi FM, Redaelli A. Mechanical properties of physiological and pathological models of collagen peptides investigated via steered molecular dynamics simulations. Journal Of Biomechanics 2008; 41:3073-3077.

40. Gautieri A, Russo A, Vesentini S, Redaelli A, Buehler MJ. Coarse-Grained Model of Collagen Molecules Using an Extended MARTINI Force Field. Journal of Chemical Theory and Computation 2010; 6:1210-1218.

41. Gautieri A, Buehler MJ, Redaelli A. Deformation rate controls elasticity and unfolding pathway of single tropocollagen molecules. Journal of the Mechanical Behavior of Biomedical Materials 2009; 2:130-137.

42. Gautieri A, Vesentini S, Redaelli A, Buehler MJ. Intermolecular slip mechanism in tropocollagen nanofibrils. International Journal of Materials Research 2009; 100:921-925.

43. Silver FH, Christiansen DL, Snowhill PB, Chen Y. Transition from viscous to elastic-based dependency of mechanical properties of self-assembled type I collagen fibers. Journal of Applied Polymer Science 2001; 79:134-142.

44. Fratzl P, Fratzlzelman N, Klaushofer K. Collagen Packing and Mineralization an X-Ray-Scattering Investigation of Turkey Leg Tendon. Biophysical Journal 1993; 64:260-266.

45. Gautieri A, Vesentini S, Montevecchi FM, Redaelli A. Mechanical properties of physiological and pathological models of collagen peptides investigated via steered molecular dynamics simulations. Journal of Biomechanics 2008; 41:3073-7.

46. Srinivasan M, Uzel SGM, Gautieri A, Keten S, Buehler MJ. Alport Syndrome mutations in type IV tropocollagen alter molecular structure and nanomechanical properties. Journal of Structural Biology 2009; 168:503-510.

47. Srinivasan M, Uzel SGM, Gautieri A, Keten S, Buehler MJ. Linking Genetics and Mechanics in Structural Protein Materials: A Case Study of an Alport Syndrome Mutation in Tropocollagen. Mathematics and Mechanics of Solids 2010; 15:755-770

48. Nakajima K, Nishi T. Nanoscience of single polymer chains revealed by nanofishing. Chemical Record 2006; 6:249258.

49. Bell Gl. Theoretical-Models for the Specific Adhesion of Cells to Cells or to Surfaces. Advances in Applied Probability 1980 12:566-567.

50. Buehler MJ, Ackbarow T. Fracture mechanics of protein materials. Materials Today 2007; 10:46-58.

51. Beck K, Chan VC, Shenoy N, Kirkpatrick A, Ramshaw JAM, Brodsky B. Destabilization of osteogenesis imperfecta collagen-like model peptides correlates with the identity of the residue replacing glycine. P. Natl. Acad. Sci. USA 2000; 97:4273-4278.
52. Bella J, Eaton M, Brodsky B, Berman HM. Crystal-structure and molecular-structure of a collagen-like peptide at 1.9 angstrom resolution. Science 1994; 266:75-81.

53. Xu Y, Persikov A, Jordan J, Brodsky B. Thermodynamic analysis of collagen-like peptides. Biophysical Journal 2000; 78:425A-425A.

54. Redaelli A, Vesentini S, Soncini M, Vena P, Mantero S, Montevecchi FM. Possible role of decorin glycosaminoglycans in fibril to fibril force transfer in relative mature tendons a computational study from molecular to microstructural level. Journal Of Biomechanics 2003; 36:1555-1569.

55. Mogilner IG, Ruderman G, Grigera JR. Collagen stability, hydration and native state. Journal of Molecular Graphics \& Modelling 2002; 21:209-213.

56. Lorenzo AC, Caffarena ER. Elastic properties, Young's modulus determination and structural stability of the tropocollagen molecule: a computational study by steered molecular dynamics. Journal Of Biomechanics 2005; 38:15271533.

57. Bhowmik R, Katti KS, Katti DR. Mechanics of molecular collagen is influenced by hydroxyapatite in natural bone. Journal of Materials Science 2007; 42:8795-8803.

58. Gautieri A, Uzel S, Vesentini S, Redaelli A, Buehler MJ. Molecular and Mesoscale Mechanisms of Osteogenesis Imperfecta Disease in Collagen Fibrils. Biophysical Journal 2009; 97:857-865.

59. Gautieri A, Vesentini S, Redaelli A, Buehler MJ. Single molecule effects of osteogenesis imperfecta mutations in tropocollagen protein domains. Protein Science 2009; 18:161-168.

60. Bhowmik R, Katti KS, Katti DR. Mechanisms of Load-Deformation Behavior of Molecular Collagen in Hydroxyapatite-Tropocollagen Molecular System: Steered Molecular Dynamics Study. Journal of Engineering Mechanics-Asce 2009; 135:413-421.

61. Gautieri A, Vesentini S, Redaelli A,Buehler MJ. Viscoelastic properties of model segments of collagen molecules. Matrix Biology 2012; 31:141-149.

62. Sun $Y$, Luo Z, Fertala A, An K. Direct quantification of the flexibility of type I collagen monomer. Biochemical And Biophysical Research Communications 2002; 295:382-386.

63. Gupta HS, Seto J, Wagermaier W, Zaslansky P, Boesecke $P$, Fratzl P. Cooperative deformation of mineral and collagen in bone at the nanoscale. P Natl Acad Sci USA 2006; 103:17741-17746.

64. Gupta HS, Wagermaier W, Zickler GA, et al. Nanoscale deformation mechanisms in bone. Nano Letters 2005; 5:2108-2111.

65. Gupta HS, Messmer P, Roschger P, Bernstorff S, Klaushofer K, Fratzl P. Synchrotron diffraction study of deformation mechanisms in mineralized tendon. Physical Review Letters 2004; 93.

66. Rauch F, Glorieux FH. Osteogenesis Imperfecta. The Lancet 2004; 363:1377-1385.

67. Buehler MJ. Nature designs tough collagen: Explaining the nanostructure of collagen fibrils. P Natl Acad Sci USA 2006; 103:12285-12290.

68. Buehler MJ. Nanomechanics of collagen fibrils under varying cross-link densities: Atomistic and continuum studies. Journal of the Mechanical Behavior of Biomedical Materials 2008; 1:59-67.

69. Orgel JPRO, Irving TC, Miller A, Wess TJ. Microfibrillar structure of type I collagen in situ. P Natl Acad Sci USA 2006; 103:9001-9005.

70. Gautieri A, Vesentini S, Redaelli A, Buehler MJ. Hierarchical Structure and Nanomechanics of Collagen Microfibrils from the Atomistic Scale Up. Nano Letters 2011; 11:757-766.

71. Chapman EW, Rodriguez F. Acrylic resin reinforcement of 
reconstituted collagen films. Polymer Engineering and Science 1977; 17:282-286.

72. Brodsky B, Ramshaw JAM. The collagen triple-helix structure. Matrix Biology 1997; 15:545-554.

73. van der Rijt JAJ, van der Werf KO, Bennink ML, Dijkstra PJ, Feijen J. Micromechanical testing of individual collagen fibrils. Macromolecular Bioscience 2006; 6:697-702.

74. Aladin DM, Cheung KM, Ngan AH, et al. Nanostructure of collagen fibrils in human nucleus pulposus and its correlation with macroscale tissue mechanics. J Orthop Res 28:497-502.

75. Espinosa HD, Zhu Y, Moldovan N. Design and operation of a MEMS-based material testing system for nanomechanical characterization. Journal Of Microelectromechanical Systems 2007; 16:1219-1231.

76. Fratzl P, Misof K, Zizak I, Rapp G, Amenitsch H, Bernstorff S. Fibrillar structure and mechanical properties of collagen. Journal of Structural Biology 1998; 122:119-122.

77. Kukreti U, Belkoff SM. Collagen fibril D-period may change as a function of strain and location in ligament. Journal of Biomechanics 2000; 33:1569-1574.

78. Ayton GS, Noid WG, Voth GA. Multiscale modeling of biomolecular systems: in serial and in parallel. Current Opinion In Structural Biology 2007; 17:192-198.

79. Noid WG, Chu JW, Ayton GS, et al. The multiscale coarse-graining method. I. A rigorous bridge between atomistic and coarsegrained models. Journal Of Chemical Physics 2008; 128.

80. Gautieri A, Mezzanzanica A, Motta A, Redealli A, Vesentini S. Atomistic modeling of water diffusion in hydrolytic biomaterials. Journal of Molecular Modeling 2011.

81. Gautieri A, Vesentini S, Redaelli A. How to predict diffusion of medium-sized molecules in polymer matrices. From atomistic to coarse grain simulations. Journal of Molecular Modeling 2010; 16:1845-1851.

82. Wang Y, Noid WG, Liu P, Voth GA. Effective force coarsegraining. Phys Chem Chem Phys 2009; 11:2002-2015.

83. Tozzini V. Coarse-grained models for proteins. Current Opinion in Structural Biology 2005; 15:144-150.

84. Marrink SJ, Risselada HJ, Yefimov S, Tieleman DP, de Vries AH. The MARTINI force field: coarse grained model for biomolecular simulations. Journal of Physical Chemistry B 2007; 111:7812-7824.

85. Monticelli L, Kandasamy SK, Periole X, Larson RG, Tieleman DP, Marrink SJ. The MARTINI coarse-grained force field: Extension to proteins. Journal of Chemical Theory and Computation 2008; 4:819-834.

86. Lopez CA, Rzepiela AJ, de Vries AH, Dijkhuizen L, Hunenberger PH, Marrink SJ. Martini Coarse-Grained Force Field:
Extension to Carbohydrates. Journal of Chemical Theory and Computation 2009; 5:3195-3210.

87. Chetwynd AP, Scott KA, Mokrab Y, Sansom MS. CGDB: a database of membrane protein/lipid interactions by coarsegrained molecular dynamics simulations. Molecular Membrane Biology 2008; 25:662-669.

88. Sansom MS, Scott KA, Bond PJ. Coarse-grained simulation: a high-throughput computational approach to membrane proteins. Biochemical Society Transactions 2008; 36:2732.

89. Treptow W, Marrink SJ, Tarek M. Gating motions in voltagegated potassium channels revealed by coarse-grained molecular dynamics simulations. Journal of Physical Chemistry B 2008; 112:3277-3282.

90. Risselada HJ, Marrink SJ. Curvature effects on lipid packing and dynamics in liposomes revealed by coarse grained molecular dynamics simulations. Physical Chemistry Chemical Physics 2009; 11:2056-2067.

91. Black SD, Mould DR. Development of hydrophobicity parameters to analyze proteins which bear posttranslational or cotranslational modifications. Anal. Biochem. 1991; 193:7282.

92. Berman HM, Westbrook J, Feng Z, et al. The Protein Data Bank. Nucleic Acids Research 2000; 28:235-242.

93. Gautieri A, Vesentini S, Montevecchi FM, Redaelli A. Mechanical properties of physiological and pathological models of collagen peptides investigated via steered molecular dynamics simulations Journal of Biomechanics 2008; 41:3073-3077. doi:10.1016/j.jbiomech. 2008.06.028.

94. Chu JW, Voth GA. Coarse-grained modeling of the actin filament derived from atomistic-scale simulations. Biophysical Journal 2006; 90:1572-1582.

95. Utiyama H, Sakato K, Ikehara K, Setsuiye T, Kurata M. Flexibility Of Tropocollagen From Sedimentation And Viscosity. Biopolymers 1973; 12:53-64.

96. Saito $\mathrm{T}$, Iso N, Mizuno H, Onda N, Yamato H, Odashima H. Semi-Flexibility Of Collagens In Solution. Biopolymers 1982; 21:715-728.

97. Nestler FHM, Hvidt S, Ferry JD, Veis A. Flexibility Of Collagen Determined From Dilute-Solution Viscoelastic Measurements. Biopolymers 1983; 22:1747-1758.

98. Bustamante C, Marko JF, Siggia ED, Smith S. Entropic Elasticity Of Lambda-Phage Dna. Science 1994; 265:15991600.

99. Buehler MJ, Wong SY. Entropic elasticity controls nanomechanics of single tropocollagen molecules. Biophysical Journal 2007; 93:37-43. 